Bull. Soc. math. France

131 (1), 2003, p. 23-39

\title{
ENSEMBLES DE TORSION NULLE DES APPLICATIONS DÉVIANT LA VERTICALE
}

\author{
PAR Sylvain CROvisier
}

\begin{abstract}
RÉSUmÉ. - Nous définissons la notion d'ensemble bien ordonné de torsion nulle pour les applications déviant la verticale. Contrairement aux études variationnelles de [14] et [1], nous proposons une approche topologique. On retrouve pour ces ensembles un grand nombre de propriétés des ensembles bien ordonnés décrites dans [11]. En reprenant un argument de G. Hall [7], nous montrons en particulier que pour tout nombre de rotation, il existe un ensemble bien ordonné de torsion nulle.

AbStract (Twist-free sets of twist maps). - We give the definition of twist-free ordered set for twist maps. Unlike the variational studies in [14] and [1], we propose a topological approach. Many properties of the ordered sets described in [11] are again satisfied by those sets. From an argument by G. Hall [7], we show in particular that there exists a twist-free ordered set for any rotation number.
\end{abstract}

\section{Introduction, rappels, notations}

On considère le tore $\mathbb{T}^{1}=\mathbb{R} / \mathbb{Z}$, l'anneau $\mathbb{A}=\mathbb{T}^{1} \times \mathbb{R}$, son revêtement universel $\widetilde{\mathbb{A}}=\mathbb{R}^{2}$, munis de leur orientation usuelle, ainsi que l'application $\pi: \widetilde{\mathbb{A}} \rightarrow \mathbb{A}$. Le relevé d'un point $z=(x, y) \in \mathbb{A}$ sera noté $\tilde{z}=(\tilde{x}, y)$. Si $X \subset \mathbb{A}$, nous noterons $\tilde{X}=\pi^{-1}(X)$.

Texte reçu le 25 juin 2001, accepté le 22 avril 2002

Sylvain Crovisier, Université Paris-Sud, Département de Mathématiques, Bâtiment 425, 91405 Orsay Cedex (France) • E-mail : Sylvain.Crovisier@math.u-psud.fr

Classification mathématique par sujets (2000). - 37E40, 37E45.

Mots clefs. - Applications déviant la verticale, nombre de rotation, ensembles d'AubryMather. 
On définit les projections $p_{1}: \mathbb{A} \rightarrow \mathbb{T}^{1}, \tilde{p}_{1}: \widetilde{\mathbb{A}} \rightarrow \mathbb{R} \operatorname{par}(x, y) \mapsto x$ et $(\tilde{x}, y) \mapsto \tilde{x}$. Pour $\tilde{z}=(\tilde{x}, y) \in \widetilde{\mathbb{A}}$, nous notons

$$
D_{i}(\tilde{z})=\left\{\left(\tilde{x}, y^{\prime}\right), y^{\prime} \leq y\right\}, \quad D_{s}(\tilde{z})=\left\{\left(\tilde{x}, y^{\prime}\right), y^{\prime} \geq y\right\} \quad \text { et } \quad D(\tilde{z})=\tilde{p}_{1}^{-1}(\tilde{x}) .
$$

L'angle entre deux vecteurs non nuls $v, w$ d'un même espace tangent $\mathrm{T}_{z} \mathbb{A}$ ou $\mathrm{T}_{\tilde{z}} \widetilde{\mathbb{A}}$, sera paramétré par le cercle orienté $\mathbb{R} / \mathbb{Z}$. Si $Y$ est une partie d'un espace topologique, nous noterons $\operatorname{Adh}(Y), \operatorname{Int}(Y)$ et $\operatorname{Fr}(Y)$ son adhérence son intérieur et sa frontière.

Une application déviant la verticale à droite (nous renvoyons à [11, ch. 1.1]) est un difféomorphisme $F$ de $\mathbb{A}$ de classe $C^{1}$ homotope à l'identité tel que pour tout relevé $\widetilde{F}$ de $F$ à $\widetilde{\mathbb{A}}$ et tout $\tilde{x} \in \mathbb{R}$, les applications

$$
y \longmapsto \tilde{p}_{1} \circ \widetilde{F}(\tilde{x}, y) \quad \text { et } \quad y \longmapsto \tilde{p}_{1} \circ \widetilde{F}^{-1}(\tilde{x}, y)
$$

soient des difféomorphismes de $\mathbb{R}$ respectivement croissant et décroissant. Nous notons $\mathrm{DV}(\mathbb{A})$ l'ensemble de ces applications et $\widetilde{\mathrm{DV}}(\mathbb{A})$ l'ensemble de leur relevés à $\widetilde{\mathbb{A}}$. On munit ces espaces de la topologie de la $C^{1}$-convergence uniforme sur les compacts.

Fixons une application $F \in \mathrm{DV}(\mathbb{A})$. Pour $z \in \mathbb{A}$, nous noterons $\mathcal{O}(z)$ son orbite par $F$ et $\widetilde{\mathcal{O}}(\tilde{z})=\pi^{-1}(\mathcal{O}(z))$.

Pour $\tilde{z} \in \widetilde{\mathbb{A}}$, on peut considérer l'ensemble (éventuellement vide) des valeurs d'adhérence dans $\mathbb{R}$ de la suite

$$
\left(\frac{\tilde{p}_{1} \circ \tilde{F}^{n}(\tilde{z})-\tilde{p}_{1}(\tilde{z})}{n}\right)_{n \in \mathbb{N} \backslash\{0\}} .
$$

C'est un ensemble qui ne dépend que de $z=\pi(\tilde{z})$, noté $\mathcal{R}(\widetilde{F}, z)$. L'union sur $z$ de ces ensembles forme l'ensemble de rotation de $\widetilde{F}$, noté $\mathcal{R}(\widetilde{F})$.

Un ensemble $\Xi \subset \mathbb{A}$ non vide, invariant par $F$, et son relevé $\widetilde{\Xi} \subset \widetilde{\mathbb{A}}$ sont dits bien ordonnés (voir [3]) si

i) $p_{1}: \Xi \rightarrow \mathbb{T}^{1}$ est injective,

ii) $\tilde{p}_{1}(\tilde{z})<\tilde{p}_{1}\left(\tilde{z}^{\prime}\right)$ implique $\tilde{p}_{1}(\widetilde{F}(\tilde{z}))<\tilde{p}_{1}\left(\widetilde{F}\left(\tilde{z}^{\prime}\right)\right)$ quels que soient $\tilde{z}, \tilde{z}^{\prime} \in \widetilde{\Xi}$.

L'ensemble $\Xi$ est alors le graphe d'une application lipschitzienne au-dessus d'un sous-ensemble de $\mathbb{T}^{1}=p_{1}(\mathbb{A})$ (voir $[8$, chap. 1]). Il existe un unique réel $\rho$, le nombre de rotation de $\widetilde{\Xi}$, tel que pour tout $\tilde{z} \in \widetilde{\Xi}$ et tout $n \in \mathbb{Z}$,

$$
-1<\tilde{p}_{1} \circ \widetilde{F}^{n}(\tilde{z})-\tilde{p}_{1}(\tilde{z})-n \rho<1 .
$$

Un ensemble bien ordonné minimal de nombre de rotation rationnel est une orbite périodique. Un ensemble bien ordonné minimal de nombre de rotation irrationnel est un graphe invariant ou un ensemble de Cantor.

Les propriétés suivantes sont alors vérifiées (voir [9], [6], [10]) :

(BO1) Si $\Xi$ est un ensemble bien ordonné, $\operatorname{Adh}(\Xi)$ est un ensemble compact bien ordonné.

TOME $131-2003-\mathrm{N}^{\mathrm{O}} 1$ 
(BO2) Si $\left(\widetilde{\Xi}_{n}\right)$ est une suite d'ensembles fermés bien ordonnés associée à une suite $\left(\widetilde{F}_{n}\right)$ d'applications déviant la verticale convergeant vers $\widetilde{F}$ dans $\widetilde{\mathrm{DV}}(\mathbb{A})$ et si $\left(\Xi_{n}\right)$ converge vers un ensemble compact $\Xi$ pour la topologie de Hausdorff, alors $\Xi$ est bien ordonné. De plus, la suite des nombres de rotation $\left(\rho_{n}\right)$ de $\left(\Xi_{n}\right)$ converge vers le nombre de rotation $\rho$ de $\Xi$.

(BO3) Pour tout $\rho \in \mathcal{R}(\widetilde{F})$, il existe un ensemble bien ordonné de nombre de rotation $\rho$ pour $\widetilde{F}$.

Remarque 1.1. - Usuellement (voir [11]), on définit l'ensemble de rotation $\mathcal{R}(\widetilde{F})$ de $\widetilde{F}$ comme l'ensemble des nombres de rotation des ensembles bien ordonnés de $\widetilde{F}$. La propriété (BO3) établit l'équivalence avec notre définition de $\mathcal{R}(\widetilde{F})$ (voir [10] et [11, chap. 1.5]).

Soit $\Xi$ un ensemble bien ordonné. L'ensemble $\alpha$-limite (resp. $\omega$-limite) de tout point $z \in \Xi$ est un ensemble bien ordonné minimal $\alpha(z)$ (resp. $\omega(z))$ inclus dans $\operatorname{Adh}(\Xi)$. L'ensemble bien ordonné $\alpha(\Xi)=\bigcup_{z} \alpha(z)$ (resp. $\omega(\Xi)=\bigcup_{z} \omega(z)$ ) est réunion d'orbites périodiques si le nombre de rotation de $\Xi$ est rationnel ou un ensemble fermé minimal sinon.

En section 2.1, nous définissons la notion d'ensemble bien ordonné de torsion nulle. Le nombre de torsion des orbites d'une application de l'anneau déviant la verticale qui préserve les aires a déjà été introduit dans [14] (amount of rotation) et [1] (twist number). Dans ces études le cadre variationnel était privilégié. Nous proposons ici de les compléter en donnant quelques propriétés topologiques. Remarquons aussi que l'hypothèse de préservation des aires est inutile ici.

Nous montrons que les propriétés (BO1-BO3) sont encore satisfaites si l'on remplace « ensemble bien ordonné » par «ensemble bien ordonné de torsion nulle ». En particulier, nous montrons le résultat suivant :

THÉORÈME 1.2. - Soit $\widetilde{F} \in \widetilde{\mathrm{DV}}(\mathbb{A})$. Si $\rho \in \mathcal{R}(\widetilde{F})$, il existe un ensemble bien ordonné pour $\widetilde{F}$ de nombre de rotation $\rho$ et de torsion nulle.

Le point essentiel, lorsque $\rho$ est rationnel, est de trouver des orbites périodiques de torsion nulle.

Dans le cadre variationnel, le nombre de torsion peut aussi être lu à partir des indices de Morse des points critiques d'une fonctionnelle associée à $F$ (voir [1]). La première orbite bien ordonnée donnée par les théorèmes d'existences de [2], [13] correspond à des minima de la fonctionnelle et est toujours de torsion nulle.

Un des ingrédients de notre démonstration s'inspire d'une idée de Hall (voir [7]) pour montrer l'existence d'orbites bien ordonnées périodiques de nombre de rotation $p / q$ lorsque $\widetilde{F}$ possède des orbites mal ordonnées périodiques de nombre de rotation $p / q$. Nous avons essayé de la reprendre et de la développer de façon rigoureuse en l'adaptant à notre situation.

BULLETIN DE LA SOCIÉtÉ MATHÉMATIQUE DE FRANCE 

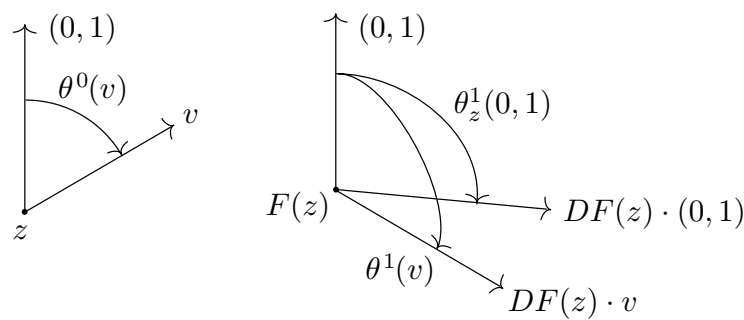

Figure 1. Définition de $\theta(v)=\theta^{1}(v)-\theta^{0}(v)$

Un grand intérêt des ensembles de torsion nulle est justifié par la remarque suivante : si $z$ possède une orbite périodique de torsion nulle, et si $z$ possède une variété invariante $\gamma$ qui est une courbe $C^{1}$, alors localement en $z, \gamma$ est un graphe au-dessus de $\mathbb{T}^{1}$. Plus précisément, si $z$ est $q$-périodique et si $v \in T_{z} M \backslash\{0\}$ est une direction invariante de $\mathrm{D}^{q}(z), v$ ne peut pas être le vecteur vertical $(0,1)$. En effet, puisque $F$ dévie la verticale à droite, l'angle entre $v$ et $\mathrm{DF}^{q}(z) \cdot v$ ne pourrait s'annuler et devrait être strictement négatif (voir section 2.2). Ceci contredirait l'hypothèse de torsion nulle. C'est le point de départ dans [4], [5] de la généralisation des langues d'Arnol'd aux applications standards dissipatives de l'anneau.

Remerciements. - Je remercie L. Guillou, P. Le Calvez et J.-C. Yoccoz pour leur lecture attentive et leurs précieuses remarques.

\section{Ensembles de torsion nulle}

Nous fixons une application $F \in \operatorname{DV}(\mathbb{A})$ et un relevé $\widetilde{F} \in \widetilde{\mathrm{DV}}(\mathbb{A})$.

2.1. Définition. - Soit $z \in \mathbb{A}$ et $v \in \mathrm{T}_{z} \mathbb{A} \backslash\{0\}$. Nous noterons $\theta^{0}(v)$ le relevé à $\mathbb{R}$ contenu dans ] - 1,0] de l'angle orienté entre le vecteur vertical $(0,1)$ et $v$. Nous notons

$$
\theta_{z}^{1}(0,1)=\theta^{0}(\mathrm{D} F(z) \cdot(0,1))
$$

et plus généralement $\theta^{1}(v)$ le relevé à $\mathbb{R}$ contenu dans $\left.] \theta_{z}^{1}(0,1)-1, \theta_{z}^{1}(0,1)\right]$ de l'angle orienté entre $(0,1)$ et $\mathrm{D} F(z) \cdot v$ (voir figure 1 ). On définit enfin

$$
\theta(v)=\theta^{1}(v)-\theta^{0}(v) .
$$

C'est un relevé à $\mathbb{R}$ de l'angle entre $v$ et $\mathrm{D} F(z) \cdot v$.

L'application $\theta: \mathrm{T}_{z} \mathbb{A} \backslash\{0\} \rightarrow \mathbb{R}$ est l'unique fonction continue qui relève l'angle entre $v$ et $\mathrm{D} F(z) \cdot v$ et pour laquelle $\theta(0,1) \in]-\frac{1}{2}, 0[$. De plus, $\theta(v)$ dépend continûment de $F$ et de $v \in \mathrm{TA}$.

TOME $131-2003-\mathrm{N}^{\mathrm{O}} 1$ 
On définit aussi

$$
\theta_{n}(v)= \begin{cases}\sum_{0 \leq k \leq n-1} \theta\left(\mathrm{D} F^{k}(z) \cdot v\right) & \text { si } n \geq 0 \\ -\theta_{-n}\left(\mathrm{D} F^{n}(z) \cdot v\right) & \text { si } n<0\end{cases}
$$

DÉfinition 2.1. - On dit qu'un ensemble invariant $X \subset \mathbb{A}$ pour une application $F \in \mathrm{DV}(\mathbb{A})$ et son relevé $\widetilde{X}$ sont de torsion nulle si pour tout $z \in X$ et tout $v \in \mathrm{T}_{z} \mathbb{A} \backslash\{0\}$,

$$
\frac{\theta_{n}(v)}{n} \underset{n \rightarrow \pm \infty}{\longrightarrow} 0
$$

Remarque 2.2. - Pour tout $z \in \mathbb{A}, v, v^{\prime} \in \mathrm{T}_{z} \mathbb{A} \backslash\{0\}$ et $n \in \mathbb{Z}$, on constate que

$$
\left|\theta_{n}(v)-\theta_{n}\left(v^{\prime}\right)\right|<\frac{1}{2}
$$

Dans cette définition, pour chaque $z \in X$, il suffit donc que (1) soit satisfait par un seul vecteur $v$ de $\mathrm{T}_{z} \mathbb{A} \backslash\{0\}$ pour l'être par tous.

2.2. Nombre de torsion des orbites périodiques. - Considérons une orbite périodique $z_{0}, z_{1}=F\left(z_{0}\right), \ldots, z_{q}=F^{q}\left(z_{0}\right)=z_{0}$. On peut définir plus généralement son nombre de torsion : c'est la quantité

$$
\lim _{n \rightarrow+\infty} \frac{\theta_{q n}(v)}{n}=\lim _{n \rightarrow-\infty} \frac{\theta_{q n}(v)}{n} .
$$

Elle ne dépend pas du vecteur $v \in \mathrm{T}_{z_{0}} \mathbb{A} \backslash\{0\}$ choisi, ni du point initial $z_{0}$ de l'orbite.

Lorsque $v$ est le vecteur vertical $(0,1)$, et $n \in \mathbb{N}$, par déviation de la verticale, $\theta_{n}(v)$ est toujours négatif.

Ainsi, le nombre de torsion est toujours négatif ou nul. $\mathrm{Si}_{\mathrm{DF}}^{q}\left(z_{0}\right)$ possède une valeur propre réelle positive (resp. négative), le nombre de torsion est un entier (resp. demi-entier non entier). Si $\mathrm{D}_{z_{0}} F^{q}$ possède une valeur propre complexe, le nombre de torsion n'est pas demi-entier mais donne l'argument des valeurs propres. Si l'on perturbe $F$ et si l'on suit continûment l'orbite de $z_{0}$, le nombre de torsion varie également de façon continue.

Remarquons que si $z$ possède une orbite périodique de torsion nulle, pour tout $v \in \mathrm{T}_{z} \mathbb{A} \backslash\{0\}$ et $n \in \mathbb{N}$, on a $1<\theta_{n}(v)<1$.

2.3. Premières propriétés. — Les ensembles bien ordonnés ont de bonnes propriétés de torsion :

Proposition 2.3. - Soit z un point d'accumulation d'un ensemble $\Xi$ bien ordonné et $v \in \mathrm{T}_{z} \mathbb{A} \backslash\{0\}$. Alors, pour tout $n \in \mathbb{Z}$,

$$
-1<\theta_{n}(v)<1 \text {. }
$$

BULletin DE LA SOCIÉtÉ MATHÉMATIQUE DE FRANCE 
Démonstration. - Nous savons que $\Xi$ est le graphe d'une application continue au-dessus de $\mathbb{T}^{1}$. Pour tous $\tilde{z}^{\prime}, \tilde{z}^{\prime \prime} \in \widetilde{\Xi}$ avec $\tilde{p}_{1}\left(\tilde{z}^{\prime}\right)<\tilde{p}_{1}\left(\tilde{z}^{\prime \prime}\right)$, l'angle entre la verticale $(0,1)$ et $\tilde{z}^{\prime \prime}-\tilde{z}^{\prime}$ possède un relevé dans $\left[-\frac{1}{2}, 0\right]$.

Nous pouvons supposer sans perdre en généralité que $\tilde{z}$ est accumulé à droite par une suite de points $\left(\tilde{z}_{k}\right)$ de $\widetilde{\Xi} \backslash\{\tilde{z}\}$ et que la suite

$$
\left(\frac{\tilde{z}_{k}-\tilde{z}}{\left\|\tilde{z}_{k}-\tilde{z}\right\|}\right)
$$

converge dans l'espace tangent unitaire $\mathrm{U}_{z} \widetilde{\mathbb{A}}$ vers un vecteur $w$. Pour tout entier $n \in \mathbb{Z}$

$$
\lim _{k} \frac{\widetilde{F}^{n}\left(\tilde{z}_{k}\right)-\widetilde{F}^{n}(\tilde{z})}{\left\|\tilde{z}_{k}-\tilde{z}\right\|}=\mathrm{D} \widetilde{F}^{n}(\tilde{z}) \cdot w .
$$

Puisque sur $\widetilde{\Xi}$, l'ordre donné par $\tilde{p}_{1}$ est préservé par $\widetilde{F}$,

$$
\forall n \in \mathbb{Z}, \quad \theta^{0}\left(\mathrm{D} \widetilde{F}^{n}(\tilde{z}) \cdot w\right) \in\left[-\frac{1}{2}, 0\right] .
$$

On en déduit,

$$
\forall n \in \mathbb{Z}, \quad-\frac{1}{2} \leq \theta_{n}(w) \leq \frac{1}{2}
$$

On termine la démonstration en utilisant (2) pour passer à un vecteur $v \in \mathrm{T}_{z} \mathbb{A}$ quelconque.

Corollaire 2.4. - Soit $\Xi$ un ensemble bien ordonné.

i) L'ensemble des points d'accumulation $\Xi_{0}$ de $\Xi$ (au sens topologique) est bien ordonné et de torsion nulle.

ii) Si $\Xi$ est de torsion nulle, $\operatorname{Adh}(\Xi)$ est bien ordonné et de torsion nulle.

Démonstration. - L'ensemble $\operatorname{Adh}(\Xi)$ est bien ordonné (propriété (BO1)). Nous lui appliquons la proposition 2.3. Les points de $\operatorname{Adh}(\Xi) \backslash \Xi$ sont des points d'accumulation de $\Xi$. Leurs orbites sont donc de torsion nulle.

Proposition 2.5. - Soit $\Xi$ un ensemble bien ordonné, alors $\Xi$ est de torsion nulle si et seulement si $\alpha(\Xi)$ et $\omega(\Xi)$ sont de torsion nulle.

Démonstration. - Si $\Xi$ est bien ordonné de torsion nulle, $\alpha(\Xi)$ et $\omega(\Xi)$ sont contenus dans $\operatorname{Adh}(\Xi)$ qui est bien ordonné et de torsion nulle d'après le corollaire précédent.

Il reste à montrer que si $\Xi$ est bien ordonné et si $\alpha(\Xi)$ et $\omega(\Xi)$ sont de torsion nulle, alors $\Xi$ est de torsion nulle.

Soit $z \in \Xi$. Alors, ou bien $\omega(z)$ est une orbite périodique, et $z \in \omega(z)$ est de torsion nulle; ou bien aucun point de $\omega(z)$ n'est isolé et on utilise la proposition 2.3 : pour tout $m \in \mathbb{N}$, il existe un voisinage ouvert $U_{m}$ de $\omega(z)$ tel que

$$
\forall z^{\prime} \in U_{m}, \forall v \in \mathrm{T}_{z^{\prime}} \mathbb{A} \backslash\{0\}, \forall k \in \mathbb{N}, \quad\left(\theta_{k}(v)<-1 \Rightarrow k>m\right) .
$$

TOME $131-2003-\mathrm{N}^{\mathrm{O}} 1$ 
Les itérés futurs de $z$ finissent par rester dans $U_{m}$. On en déduit lorsque $n$ tend vers $+\infty$

$$
-\frac{1}{m} \leq \underline{\lim } \frac{\theta_{n}(0,1)}{n} \leq \varlimsup \frac{\theta_{n}(0,1)}{n} \leq 0 .
$$

On raisonne de même en $-\infty$.

REMARque 2.6. - Lorsque $\Xi$ est bien ordonné de torsion nulle, les convergences (1) sont uniformes en $z \in \Xi$ et $v \in \mathrm{T}_{z} \mathbb{A}$ : en effet $\theta$ est une application bornée. D'autre part, $\alpha(\Xi)$ et $\omega(\Xi)$ sont soit des ensembles bien ordonnés irrationnels, soit des unions d'orbites périodiques de torsion nulle. L'application $\theta_{n}$ y est donc bornée uniformément en $n$.

COROllaire 2.7. - Tout ensemble bien ordonné $\Xi$ de nombre de rotation irrationnel est de torsion nulle.

Démonstration. — Les ensemble $\alpha(\Xi)$ et $\omega(\Xi)$ sont minimaux et donc de torsion nulle d'après la proposition 2.3. On applique ensuite la proposition 2.5.

Tout comme les ensembles bien ordonnés, les ensembles bien ordonnés de torsion nulle sont préservés par passage à la limite :

Proposition 2.8. - Soit $\left(\Xi_{k}\right)$ une suite d'ensembles fermés bien ordonnés de torsion nulle associée à une suite $\left(F_{k}\right)$ d'applications déviant la verticale. On suppose $\omega\left(\Xi_{k}\right)=\Xi_{k}$. Si la suite $\left(F_{k}\right)$ converge vers une application $F$ déviant la verticale, pour la topologie de $\mathrm{DV}(\mathbb{A})$ et si la suite $\left(\Xi_{k}\right)$ converge vers un compact de l'anneau $\Xi$, pour la distance de Hausdorff, alors $\Xi$ est bien ordonné et de torsion nulle.

Démonstration. - On sait déjà (propriété (BO2)) que $\Xi$ est bien ordonné.

Puisque $\omega\left(\Xi_{k}\right)=\Xi_{k}$, chaque point de $\Xi_{k}$ est soit une orbite périodique, soit un point d'accumulation de $\Xi_{k}$. On en déduit grâce à la proposition 2.3 et la section 2.2 que pour tout $N \in \mathbb{Z}$ et $k \in \mathbb{N}$, pour tout $z \in \Xi_{k}$ et $v \in \mathrm{T}_{z} \mathbb{A} \backslash\{0\}$,

$$
\left|\theta_{N}\left(F_{k}, v\right)\right|<1
$$

où l'on a précisé la dépendance en l'application $F_{k}$.

Par continuité de $\theta_{N}$, on obtient pour tout $z \in \Xi$ et $v \in \mathrm{TA} \backslash\{0\}$,

$$
\left|\theta_{N}(F, v)\right| \leq 1 \text {. }
$$

Ceci conclut la démonstration.

On en déduit :

Corollaire 2.9. - Si F est une application de DV(A), l'union des compacts bien ordonnés minimaux de torsion nulle est un ensemble fermé de torsion nulle. 


\section{Existence d'ensembles bien ordonnés de torsion nulle}

3.1. Démonstration du théorème 1.2. - La preuve, purement topologique, montre qu'un ensemble bien ordonné, maximal pour l'inclusion, possède toujours une orbite de torsion nulle.

La démonstration repose sur le lemme suivant :

Lemme 3.1. - Soit $\Xi$ un ensemble bien ordonné de $F \in \mathrm{DV}(\mathbb{A})$ formé de deux orbites périodiques bien ordonnées de nombre de rotation $p / q, \mathcal{O}\left(z_{0}\right)$ et $\mathcal{O}\left(z_{0}^{\prime}\right)$, éventuellement confondues et qui ne sont pas de torsion nulle. Nous supposons que les points $z_{0}$ et $z_{0}^{\prime}$ possèdent des relevés $\tilde{z}_{0}$ et $\tilde{z}_{0}^{\prime}$ tels que $\tilde{p}_{1}\left(\tilde{z}_{0}\right)<\tilde{p}_{1}\left(\tilde{z}_{0}^{\prime}\right)$ soient deux points consécutifs de $\tilde{p}_{1}(\widetilde{\Xi})$. Alors il existe une troisième orbite périodique bien ordonnée de nombre de rotation $p / q, \mathcal{O}\left(z_{0}^{\prime \prime}\right)$ et un relevé $\tilde{z}_{0}^{\prime \prime}$ de $z_{0}^{\prime \prime}$ tels que

i) $\tilde{p}_{1}\left(\tilde{z}_{0}\right)<\tilde{p}_{1}\left(\tilde{z}_{0}^{\prime \prime}\right)<\tilde{p}_{1}\left(\tilde{z}_{0}^{\prime}\right)$,

ii) $\Xi \cup \mathcal{O}\left(z_{0}^{\prime \prime}\right)$ est bien ordonné.

Nous passons à la démonstration du théorème.

Pour tout $\rho \in \mathcal{R}(\widetilde{F})$, il existe pour $F$ un ensemble bien ordonné $\Xi$ minimal de nombre de rotation $\rho$ (propriété (BO3)). Lorsque $\rho$ est irrationnel, la conclusion suit du corollaire 2.7 .

Nous traitons les autres cas par l'absurde : supposons que $\rho$ soit un rationnel $\rho=p / q$ et que $F$ ne possède pas d'orbite périodique bien ordonnée de nombre de rotation $p / q$ et de torsion nulle. L'ensemble $\Xi$ est une orbite périodique bien ordonnée de nombre de rotation $p / q, \mathcal{O}(z)$. On fixe un relevé $\tilde{z}(0)$ de $z$ et on pose $\widetilde{\Xi}(0)=\tilde{\Xi}=\widetilde{\mathcal{O}}(z(0))$. Grâce au lemme précédent, on construit une suite d'orbites périodiques bien ordonnées de nombre de rotation $p / q$ (de torsion non nulle par hypothèse), $(\widetilde{\mathcal{O}}(z(n)))$, distinctes, et une suite d'ensembles bien ordonnés $(\widetilde{\Xi}(n))$ tels que pour tout $n \geq 0$,

i) $\widetilde{\Xi}(n+1)=\widetilde{\Xi}(n) \cup \widetilde{\mathcal{O}}(z(n+1))$,

ii) $\tilde{p}_{1}(\tilde{z}(0))<\tilde{p}_{1}(\tilde{z}(n+1))$ sont deux point consécutifs de $\tilde{p}_{1}(\widetilde{\Xi}(n+1))$.

Soit $\Xi(\infty)$ une valeur d'adhérence de la suite $(\Xi(n))=(\pi(\widetilde{\Xi}(n)))$. D'après la propriété (BO2), c'est un ensemble bien ordonné. Nous considérons alors $\tilde{z}^{\prime}=\lim _{n} \tilde{z}(n)$. Notons que $z^{\prime}=\pi\left(\tilde{z}^{\prime}\right)$ est un point d'accumulation de $\Xi(\infty)$. L'orbite $\mathcal{O}\left(z^{\prime}\right)$ est donc bien ordonnée de nombre de rotation $p / q$ et de torsion nulle. Ceci est une contradiction.

3.2. Un lemme de topologie du plan. - Nous rappelons qu'un arc est une application injective de $[0,1]$ dans $\widetilde{\mathbb{A}}$. Un disque topologique fermé sera une partie de $\widetilde{\mathbb{A}}$ homéomorphe à

$$
\left\{(x, y) \in \mathbb{R}^{2}, x^{2}+y^{2} \leq 1\right\} .
$$

TOME $131-2003-\mathrm{N}^{\mathrm{O}} 1$ 


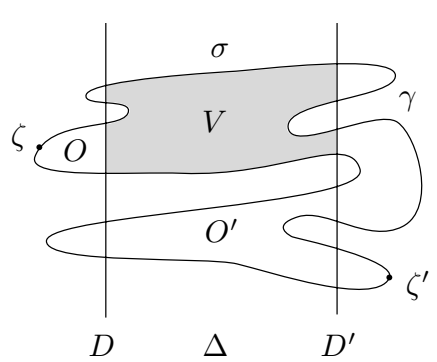

(a)

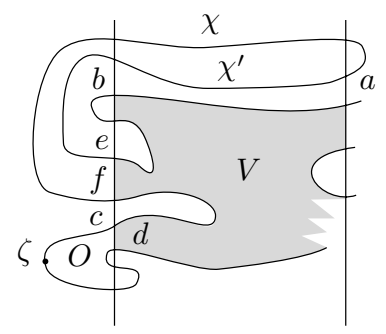

(b)

FiguRE 2. Intersection $U \cap \Delta$

Une courbe de Jordan sera une partie de $\widetilde{\mathbb{A}}$ homéomorphe à $\mathbb{T}^{1}$. La composante connexe bornée de son complémentaire sera appelée un domaine de Jordan. Les courbes de Jordan seront orientées positivement. Si $x$ et $y$ sont deux points distincts d'un même arc ou d'une même courbe de Jordan $\gamma$, nous désignerons $\operatorname{par}(x, y)_{\gamma}$ (resp. $\left.[x, y]_{\gamma}\right)$ l'arc ouvert (resp. fermé) issu de $x$ et aboutissant en $y$ le long de $\gamma$.

La réunion d'une courbe de Jordan et du domaine de Jordan correspondant forme un disque topologique fermé. Nous utiliserons également les résultats suivants dûs à B. de Kérékjárto (voir [12]) :

i) Toute composante connexe de l'intersection de deux domaines de Jordan est encore un domaine de Jordan.

ii) Soient $U$ et $U^{\prime}$ deux domaines de Jordan tels que $U \cap U^{\prime} \neq \varnothing, U \not \subset U^{\prime}$, et $U^{\prime} \not \subset U$. Si $V$ est une composante connexe de $U \cap U^{\prime}$, et si $\gamma=\operatorname{Fr}(U)$, $\gamma^{\prime}=\operatorname{Fr}\left(U^{\prime}\right)$ et $\sigma=\operatorname{Fr}(V)$ alors, les composantes connexes de $\sigma \backslash \gamma$ forment une famille au plus dénombrable d'arcs de $\gamma^{\prime}$ de la forme $(x, y)_{\gamma^{\prime}}$ où $x$ et $y$ sont des points distincts de $\gamma \cap \gamma^{\prime} \cap \sigma$.

iii) Soit $(x, y)_{\gamma^{\prime}}$ une composante de $\sigma \backslash \gamma$. Alors, $(x, y)_{\gamma} \cup(x, y)_{\gamma^{\prime}} \cup\{x, y\}$ est une courbe de Jordan bordant un domaine de Jordan $O$. On dira que $O$ est associée à $(x, y)_{\gamma^{\prime}}$.

iv) Les composantes connexes de $U \backslash \operatorname{Adh}(V)$ sont exactement les domaines $O$ construits ainsi.

Pour démontrer le lemme 3.1, nous aurons besoin d'un lemme de topologie du plan (voir figure $2(\mathrm{a})$ ).

Lemme 3.2. - Considérons la bande $\Delta=\{(\tilde{x}, y), 0 \leq \tilde{x} \leq 1\}$ dans le plan bordée par les droites $D=\{(0, y), y \in \mathbb{R}\}, D^{\prime}=\{(1, y), y \in \mathbb{R}\}$ et $U$ un domaine de Jordan bordé par $\gamma$. Supposons que $\gamma$ possède deux points $\zeta$ et $\zeta^{\prime}$ respectivement à gauche et à droite de $\Delta\left(\tilde{p}_{1}(\zeta)<0<1<\tilde{p}_{1}\left(\zeta^{\prime}\right)\right)$. Alors, il existe une composante $V$ de $U \cap \operatorname{Int}(\Delta)$, bordée par une courbe de Jordan $\sigma$, qui sépare $\zeta$ de $\zeta^{\prime}$ dans $U$ : la composante $O\left(\right.$ resp. $\left.O^{\prime}\right)$ de $U \backslash \operatorname{Adh}(V)$ qui 
contient $\zeta\left(\right.$ resp. $\left.\zeta^{\prime}\right)$ dans son adhérence est associée à une composante de $\sigma \backslash \gamma$ incluse dans $D$ (resp. $\left.D^{\prime}\right)$.

Démonstration. — Tout d'abord, on remarque que l'on peut plonger $\widetilde{\mathbb{A}}$ dans le disque ouvert $B(0,1)$ du plan $\mathbb{R}^{2}$ au moyen de l'application

$$
(\tilde{x}, y) \longmapsto\left(\frac{\tilde{x}}{\left(1+\tilde{x}^{2}+y^{2}\right)^{1 / 2}}, \frac{y}{\left(1+\tilde{x}^{2}+y^{2}\right)^{1 / 2}}\right)
$$

Définissons les points $A=(0,1)$ et $B=(0,-1)$ de $\mathbb{R}^{2}$. On considère alors $\Delta$ comme un domaine de Jordan de frontière $D \cup D^{\prime} \cup\{A, B\}$ dans $\mathbb{R}^{2}$. Le résultat de Kérékjárto s'applique donc aux ouverts $U$ et $\operatorname{Int}(\Delta)$.

a) Nous appelons $\Sigma$ l'ensemble des composantes de $\gamma \cap \operatorname{Int}(\Delta)$ joignant $D$ à $D^{\prime}$. Par hypothèse, $\Sigma$ est non vide et possède au moins deux éléments. Chaque élément de $\Sigma$ a un diamètre supérieur ou égal à 1 . Par compacité de $\gamma, \Sigma$ est donc fini.

Si $(a, b)_{\gamma}$ est dans $\Sigma$, alors $\{a, b\}$ possède exactement un point dans chaque droite $D$ et $D^{\prime}$. On en déduit que $\Sigma$ est réunion disjointe de deux ensembles $\Sigma_{g}$ et $\Sigma_{d}$ : l'arc $(a, b)_{\gamma}$ appartient à $\Sigma_{d}\left(\right.$ resp. $\left.\Sigma_{g}\right)$ si et seulement si $a \in D$ (resp. $a \in D^{\prime}$ ).

Chaque arc $\chi$ de $\Sigma$ est dans la frontière d'une unique composante $V$ de $U \cap \operatorname{Int}(\Delta)$. Réciproquement, et par connexité, toute composante $V$ de $U \cap$ $\operatorname{Int}(\Delta)$ joignant $D$ à $D^{\prime}$ contient dans sa frontière exactement un arc de chaque ensemble $\Sigma_{g}$ et $\Sigma_{d}$

b) L'arc $\left(\zeta, \zeta^{\prime}\right)_{\gamma}$ contient $n$ éléments de $\Sigma_{g}$ et $n+1$ éléments de $\Sigma_{d}$; l'arc $\left(\zeta^{\prime}, \zeta\right)_{\gamma}$ contient $m$ éléments de $\Sigma_{d}$ et $m+1$ éléments de $\Sigma_{g}$ avec $n, m \in \mathbb{N}$. On en déduit qu'il existe une composante $V$ de $U \cap \operatorname{Int}(\Delta)$ bordée par une courbe de Jordan $\sigma$ contenant un arc $\sigma_{d} \subset\left(\zeta, \zeta^{\prime}\right)_{\gamma}$ de $\Sigma_{d}$ et un arc $\sigma_{g} \subset\left(\zeta^{\prime}, \zeta\right)_{\gamma}$ de $\Sigma_{g}$.

c) Nous montrons que $V$ convient. Nous notons $(a, b)_{\sigma}=\sigma_{g}$. La composante $O$ de $U \backslash \operatorname{Adh}(V)$ qui contient $\zeta$ dans son adhérence est bordée par un arc $(c, d)_{\gamma} \cup[d, c]_{D}$. Soit, s'il en existe, $\chi \in \Sigma$ un arc contenu dans $(b, c)_{\gamma} \subset\left(\zeta^{\prime}, \zeta\right)_{\gamma}$. Alors, $\chi$ est dans la frontière d'une unique composante $W$ de $U \backslash \operatorname{Adh}(V)$ distincte de $O$. La frontière de $W$ est de la forme $(e, f)_{\gamma} \cup[e, f]_{\sigma}$ avec $(e, f)_{\gamma} \subset$ $(b, c)_{\gamma}$ et $[e, f]_{\sigma} \subset D \cup D^{\prime}$. On en déduit que $(e, f)_{\gamma}$ contient l'autre arc $\chi^{\prime}$ de $\Sigma$ contenu dans la frontière de $W$ (voir figure $2(\mathrm{~b})$ ).

Ainsi $(b, c)_{\gamma}$ contient un nombre pair d'arc de $\Sigma$. Puisque $\zeta$ est à gauche de $D$ et $b \in D,(b, \zeta)_{\gamma}$ contient également un nombre pair d'arcs de $\Sigma$. Finalement, $(c, \zeta)_{\gamma}$ contient un nombre pair d'arcs de $\Sigma$. Puisque $\zeta$ est à gauche de $D$ et $c \in D \cup D^{\prime}$, on obtient $c \in D$. Par conséquent $O$ est rattachée à $V$ par un arc $[c, d]$, contenu dans $D$. Le même raisonnement s'applique à $O^{\prime}$, la composante de $U \backslash \operatorname{Adh}(V)$ qui contient $\zeta^{\prime}$.

TOME $131-2003-\mathrm{N}^{\mathrm{O}} 1$ 


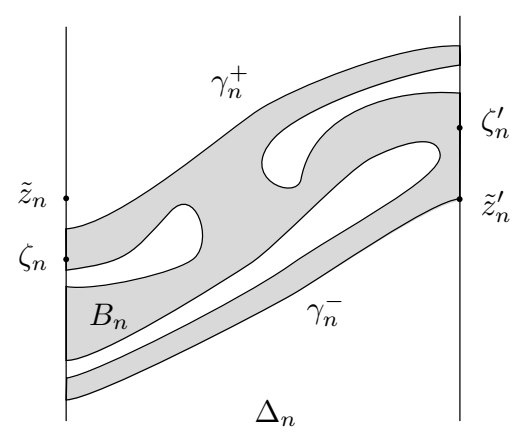

Figure 3. La région $B_{n}$

3.3. Démonstration du lemme 3.1. - On choisit $p$ et $q$ premiers entre eux; la période de $\mathcal{O}\left(z_{0}\right)$ et $\mathcal{O}\left(z_{0}^{\prime}\right)$ est alors $q$. On pose

$$
\tilde{z}_{n}=\widetilde{F}^{n}\left(\tilde{z}_{0}\right) \text { et } \quad \tilde{z}_{n}^{\prime}=\widetilde{F}^{n}\left(\tilde{z}_{0}^{\prime}\right) \text {. }
$$

Nous définissons pour chaque $n \in \mathbb{Z}$ la région

$$
\Delta_{n}=\left\{(\tilde{x}, y), \tilde{p}_{1}\left(\tilde{z}_{n}\right) \leq \tilde{x} \leq \tilde{p}_{1}\left(\tilde{z}_{n}^{\prime}\right)\right\} .
$$

Première étape : construction des régions $B_{n}$. - On exhibe pour $n \geq 1$ une composante connexe $B_{n}$ de

$$
\left\{\tilde{z}, \forall k, 0 \leq k \leq n, \widetilde{F}^{k-n}(\tilde{z}) \in \Delta_{k}\right\}
$$

qui vérifie les conditions suivantes (voir figure 3 ) :

i) $B_{n}$ est un disque topologique fermé. Le bord de $B_{n}$ est une courbe de Jordan $\gamma_{n}$.

ii) Il existe deux points $\zeta_{n} \in \gamma_{n} \cap D_{i}\left(\tilde{z}_{n}\right)$ et $\zeta_{n}^{\prime} \in \gamma_{n} \cap D_{s}\left(\tilde{z}_{n}^{\prime}\right)$ tels que les composantes de $\left[\zeta_{n}, \zeta_{n}^{\prime}\right]_{\gamma_{n}} \cap \operatorname{Int}\left(\Delta_{n}\right)$ (resp. $\left.\left[\zeta_{n}^{\prime}, \zeta_{n}\right]_{\gamma_{n}} \cap \operatorname{Int}\left(\Delta_{n}\right)\right)$ forment une famille au plus dénombrable d'arcs $(\eta, \xi)_{\gamma_{n}}$ de la forme $\widetilde{F}^{n}(I)$ où $I$ est un intervalle ouvert de $D_{i}\left(\tilde{z}_{0}^{\prime}\right)$ (resp. $\left.D_{s}\left(\tilde{z}_{0}\right)\right)$. De plus, $\eta$ et $\xi$ sont des points de $D_{i}\left(\tilde{z}_{n}\right)$ ou de $D_{s}\left(\tilde{z}_{n}^{\prime}\right)$.

iii) $\left[\zeta_{n}^{\prime}, \zeta_{n}\right]_{\gamma_{n}}\left(\right.$ resp. $\left[\zeta_{n}, \zeta_{n}^{\prime}\right]_{\gamma_{n}}$ ) possède exactement un $\operatorname{arc} \gamma_{n}^{+}=(\eta, \xi)_{\gamma_{n}}$ (resp. $\left.\gamma_{n}^{-}=(\xi, \eta)_{\gamma_{n}}\right)$ de la forme $\widetilde{F}^{n}\left(I_{n}^{+}\right)\left(\operatorname{resp} . \widetilde{F}^{n}\left(I_{n}^{-}\right)\right)$contenu dans $\operatorname{Int}\left(\Delta_{n}\right)$, où $I_{n}^{+}$(resp. $\left.I_{n}^{-}\right)$est un intervalle ouvert de $D_{s}\left(\tilde{z}_{0}\right)$ (resp. $\left.D_{i}\left(\tilde{z}_{0}^{\prime}\right)\right)$ tel que $\{\eta, \xi\}$ contienne à la fois un point de $D_{i}\left(\tilde{z}_{n}\right)$ et un point de $D_{s}\left(\tilde{z}_{n}^{\prime}\right)$. De plus, $\eta \in D_{s}\left(\tilde{z}_{n}^{\prime}\right), \xi \in D_{i}\left(\tilde{z}_{n}\right)$.

iv) $\gamma_{n} \cap \operatorname{Fr}\left(\Delta_{n}\right)$ est inclus dans $\mathrm{D}_{i}\left(\tilde{z}_{n}\right) \cup \mathrm{D}_{s}\left(\tilde{z}_{n}^{\prime}\right)$. Le point $\zeta_{n}$ (resp. $\left.\zeta_{n}^{\prime}\right)$ appartient à un segment ouvert de $\mathrm{D}_{i}\left(\tilde{z}_{n}\right)\left(\right.$ resp. $\left.\mathrm{D}_{s}\left(\tilde{z}_{n}^{\prime}\right)\right)$ inclus dans $\gamma_{n}$.

La construction se fait par récurrence sur $n \geq 1$ :

a) On pose

$$
B_{1}=\widetilde{F}\left(\Delta_{0}\right) \cap \Delta_{1} .
$$

BULLETIN DE LA SOCiÉTÉ MATHÉMATIQUe DE FRANCE 


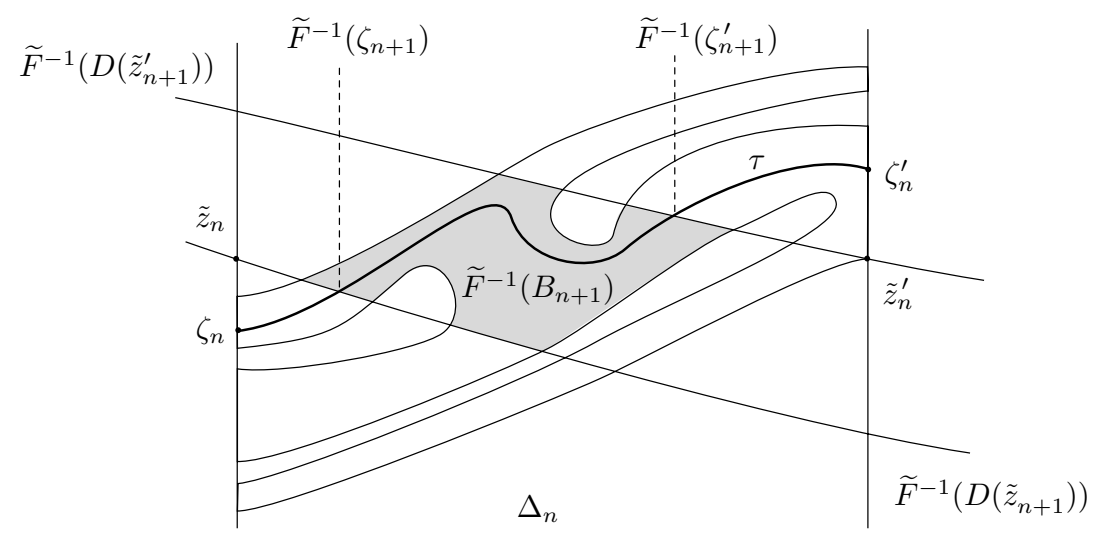

FiguRE 4. Construction de $B_{n+1}$

Par déviation de la verticale à droite, les propriétés i) à iv) sont faciles à vérifier pour $B_{1}$. Nous fixons des points $\zeta_{1}$ et $\zeta_{1}^{\prime}$.

Nous construisons maintenant $B_{n+1}$ à partir de $B_{n}$ pour $n \geq 1$ (figure 4 ).

Par déviation de la verticale, $\zeta_{n}$ est en-dessous du graphe $\widetilde{F}^{-1}\left(D\left(\tilde{z}_{n+1}\right)\right)$ et $\zeta_{n}^{\prime}$ au-dessus du graphe $\widetilde{F}^{-1}\left(D\left(\tilde{z}_{n+1}^{\prime}\right)\right)$. Nous appliquons alors le lemme 3.2 à la bande $\widetilde{F}^{-1}\left(\Delta_{n+1}\right)$, au domaine $\operatorname{Int}\left(B_{n}\right)$ et aux points $\zeta_{n}, \zeta_{n}^{\prime}$ pour construire un domaine de Jordan $V$. On définit

$$
B_{n+1}=\widetilde{F}(\operatorname{Adh}(V)) .
$$

Nous notons encore $O$ et $O^{\prime}$ les composantes de $\operatorname{Int}\left(B_{n}\right) \backslash \operatorname{Adh}(V)$ qui contiennent $\zeta_{n}$ et $\zeta_{n}^{\prime}$ dans leur fontière.

b) Il reste à vérifier pour $B_{n+1}$ les propriétés ii) à iv) :

Remarquons tout d'abord que par déviation de la verticale, $\gamma_{n+1}$ ne rencontre ni $D_{s}\left(\tilde{z}_{n+1}\right) \backslash\left\{\tilde{z}_{n+1}\right\}$ ni $D_{i}\left(\tilde{z}_{n+1}^{\prime}\right) \backslash\left\{\tilde{z}_{n+1}^{\prime}\right\}$. La composante $O$ (resp. $O^{\prime}$ ) est associée à une composante de $\widetilde{F}^{-1}\left(\gamma_{n+1}\right) \backslash \gamma_{n}$ qui est un segment ouvert de $\widetilde{F}^{-1}\left(D_{i}\left(\tilde{z}_{n+1}\right)\right)$ (resp. $\left.\widetilde{F}^{-1}\left(D_{s}\left(\tilde{z}_{n+1}^{\prime}\right)\right)\right)$. On choisit sur ce segment un point $\widetilde{F}^{-1}\left(\zeta_{n+1}\right)$ (resp. $\left.\widetilde{F}^{-1}\left(\zeta_{n+1}^{\prime}\right)\right)$ arbitraire. Ceci montre le iv).

Les composantes connexes de $\gamma_{n+1} \backslash\left(D_{i}\left(\tilde{z}_{n+1}\right) \cup D_{s}\left(\tilde{z}_{n+1}^{\prime}\right)\right)$ forment une famille au plus dénombrable d'arcs $(\eta, \xi)_{\gamma_{n+1}}$ de $\widetilde{F}\left(\gamma_{n}\right)$ contenus dans $\operatorname{Int}\left(\Delta_{n+1}\right)$, d'après le résultat de Kérékjárto. Par déviation de la verticale, les images de $D_{i}\left(\tilde{z}_{n}\right)$ et $D_{s}\left(\tilde{z}_{n}^{\prime}\right)$ n'intersectent pas $\operatorname{Int}\left(\Delta_{n+1}\right)$. L'arc $(\eta, \xi)_{\gamma_{n+1}}$ est donc de la forme $\widetilde{F}\left(\widetilde{F}^{n}(I)\right)$ où $I$ est un intervalle ouvert de $D_{s}\left(\tilde{z}_{0}\right)$ ou de $D_{i}\left(\tilde{z}_{0}^{\prime}\right)$. Bien sûr, $\eta$ et $\xi$ sont des points de $D_{i}\left(\tilde{z}_{n+1}\right) \cup D_{s}\left(\tilde{z}_{n+1}^{\prime}\right)$.

TOME $131-2003-\mathrm{N}^{\mathrm{O}} 1$ 

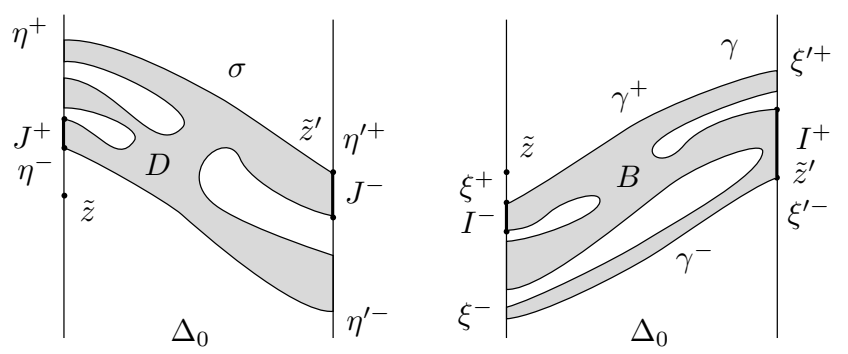

Figure 5. Les régions $D$ et $B$

On peut construire un $\operatorname{arc} \tau$ dans $B_{n}$ issu de $\zeta_{n}$, aboutissant en $\zeta_{n}^{\prime}$ et passant par $\widetilde{F}^{-1}\left(\zeta_{n+1}\right)$ et $\widetilde{F}^{-1}\left(\zeta_{n+1}^{\prime}\right)$. On peut supposer que

$$
\left(\zeta_{n}, \widetilde{F}^{-1}\left(\zeta_{n+1}\right)\right)_{\tau}, \quad\left(\widetilde{F}^{-1}\left(\zeta_{n+1}\right), \widetilde{F}^{-1}\left(\zeta_{n+1}^{\prime}\right)\right)_{\tau} \quad \text { et } \quad\left(\widetilde{F}^{-1}\left(\zeta_{n+1}\right), \zeta_{n}^{\prime}\right)_{\tau}
$$

sont respectivement inclus dans $O, V$ et $O^{\prime}$. L'arc $\tau$ sépare $\Delta_{n}$. On obtient ainsi que les arcs de la forme $\widetilde{F}\left(\widetilde{F}^{n}(I)\right)=(\eta, \xi)_{\gamma_{n+1}}$ inclus dans $\left(\zeta_{n+1}^{\prime}, \zeta_{n+1}\right)_{\gamma_{n+1}}$ (resp. $\left.\left(\zeta_{n+1}, \zeta_{n+1}^{\prime}\right)_{\gamma_{n+1}}\right)$ sont images par $\widetilde{F}$ de sous-arcs de $\left(\zeta_{n}^{\prime}, \zeta_{n}\right)_{\gamma_{n}}$ (resp. $\left.\left(\zeta_{n}, \zeta_{n}^{\prime}\right)_{\gamma_{n}}\right)$. Le segment $I$ est donc un intervalle ouvert de $D_{s}\left(\tilde{z}_{0}\right)\left(\right.$ resp. $\left.D_{i}\left(\tilde{z}_{0}^{\prime}\right)\right)$. Ceci termine la démonstration du ii).

Nous avons déjà remarqué lors de la démonstration du lemme 3.2 que puisque $B_{n+1}$ est connexe, inclus dans $\Delta_{n+1}$ et joint $D\left(\tilde{z}_{n+1}\right)$ à $D\left(\tilde{z}_{n+1}^{\prime}\right)$, il existe exactement un $\operatorname{arc}(\eta, \xi)_{\gamma_{n+1}}=\widetilde{F}^{n+1}(I)$ contenu dans $\left(\zeta_{n+1}, \zeta_{n+1}^{\prime}\right)_{\gamma_{n+1}}$ joignant $D\left(\tilde{z}_{n+1}\right)$ à $D\left(\tilde{z}_{n+1}^{\prime}\right)$. On obtient également $\eta \in D_{s}\left(\tilde{z}_{n+1}^{\prime}\right)$ et $\xi \in D_{i}\left(\tilde{z}_{n+1}\right)$. On montre une propriété analogue avec $\left(\zeta_{n+1}^{\prime}, \zeta_{n+1}\right)_{\gamma_{n+1}}$.

Deuxième étape : construction d'une courbe d'indice -1. - Nous allons étudier $D=\widetilde{F}^{-q}\left(B_{q}\right)$ et $B=B_{q}-(p, 0)$, qui sont des disques topologiques fermés inclus dans $\Delta_{0}$, images l'un de l'autre par le difféomorphisme $\widetilde{G}=\widetilde{F}^{q}-(p, 0)$, et bordés par des courbes $\sigma$ et $\gamma$ respectivement.

a) Les propriétés de $B_{q}$ se transposent à $B$. On définit

$$
\gamma^{+}=\gamma_{q}^{+}-(p, 0) \quad \text { et } \quad \gamma^{-}=\gamma_{q}^{-}-(p, 0) .
$$

Il existe pour $D$ des propriétés similaires (voir figure 5 ) :

- En appliquant $\widetilde{F}^{-q}$ à $\gamma_{q}$, on obtient que les composantes connexes qui composent $\sigma \cap \operatorname{Int}\left(\Delta_{0}\right)$ sont des arcs la forme $\widetilde{G}^{-1}(I)$, où $I$ est un intervalle ouvert de $D_{i}\left(\tilde{z}_{0}\right)$ ou $D_{s}\left(\tilde{z}_{0}^{\prime}\right)$.

- Soit $I$ la composante de $\gamma_{q} \backslash \widetilde{F}\left(\gamma_{q-1}\right)$ qui contient $\zeta_{q}$. C'est un intervalle ouvert de $D_{i}\left(\tilde{z}_{q}\right)$. D'après la propriété ii), $\left(\eta^{-}, \eta^{\prime-}\right)_{\sigma}=\widetilde{F}^{-q}(I)$ est un arc qui joint $D_{s}\left(\tilde{z}_{0}\right)$ à $D_{i}\left(\tilde{z}_{0}^{\prime}\right)$. Il existe également un $\operatorname{arc}\left(\eta^{\prime+}, \eta^{+}\right)_{\sigma}=\widetilde{F}^{-q}\left(I^{\prime}\right)$, où $I^{\prime}$ est la composante connexe de $\gamma_{q} \backslash \widetilde{F}\left(\gamma_{q-1}\right)$ qui contient $\zeta_{q}^{\prime}$. Là encore $\left(\eta^{\prime+}, \eta^{+}\right)_{\sigma}$ 

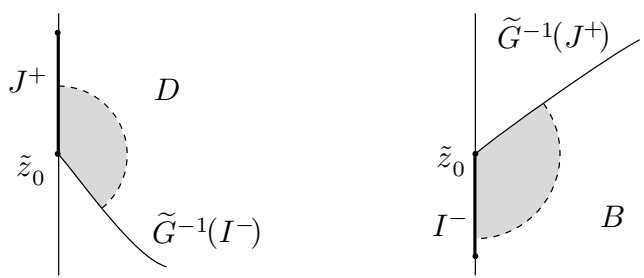

Figure 6 . Modification de $D$ et $B$ près de $\tilde{z}_{0}$

joint $D_{i}\left(\tilde{z}_{0}^{\prime}\right)$ à $D_{s}\left(\tilde{z}_{0}\right)$. Ces arcs sont uniques puisque $\operatorname{Int}(D) \subset \Delta_{0}$ est connexe. On note

$$
I^{-}=I-(p, 0), \quad I^{+}=I^{\prime}-(p, 0) .
$$

- $\left(\eta^{+}, \eta^{-}\right)_{\sigma}$ (resp. $\left.\left(\eta^{\prime-}, \eta^{+}\right)_{\sigma}\right)$ contient $J^{+}\left(\right.$resp. $\left.J^{-}\right)$, intervalle ouvert de $D_{s}\left(\tilde{z}_{0}\right)$ (resp. $\left.D_{i}\left(\tilde{z}_{0}^{\prime}\right)\right)$ tel que $\widetilde{G}\left(J^{+}\right)=\gamma^{+}$et $\widetilde{G}\left(J^{-}\right)=\gamma^{-}$.

b) Dans le cas où $\sigma$ (et $\gamma$ ) rencontre $\tilde{z}_{0}$, nous modifions $\sigma$ (et $\left.\gamma\right)$ près de $\tilde{z}_{0}$ : d'après ce que nous avons vu, $\eta^{-}=\xi^{+}=\tilde{z}_{0}$. Au voisinage de $\tilde{z}_{0}, \sigma$ (resp. $\gamma$ ) est la réunion de deux chemins $J^{+} \cup \tilde{G}^{-1}\left(I^{-}\right) \cup\left\{\tilde{z}_{0}\right\}$ (resp. $\widetilde{G}\left(J^{+}\right) \cup I^{-} \cup\left\{\tilde{z}_{0}\right\}$ ). Le nombre de torsion de $\tilde{z}_{0}$ est donc dans $\left[-\frac{1}{2}, 0[\right.$.

On enlève maintenant à $B$ une boule euclidienne ouverte $\operatorname{Int}\left(B\left(\tilde{z}_{0}, r\right)\right)$ de rayon $r>0$ assez petit. On procède de la même façon au voisinage de $\tilde{z}_{0}^{\prime}$ dans le cas où $\gamma$ rencontre $\tilde{z}_{0}$. On obtient ainsi un nouveau disque topologique fermé $\hat{B} \subset B$, bordé par une courbe de Jordan $\hat{\gamma}$ et image par $\widetilde{G}$ d'un disque $\hat{D} \subset D$, bordé par une courbe de Jordan $\hat{\sigma}$. Ils sont tous deux contenus dans $\Delta_{0}$ (voir figure 6$)$.

c) Si $\tilde{z}$ est un point fixe de $\widetilde{G}$ dans $D$, l'ensemble $\mathcal{O}(z) \cup \mathcal{O}\left(z_{0}\right)$ est bien ordonné. Le point $\tilde{z}_{0}$ ne peut être accumulé par des points fixes de $\widetilde{G}$ contenus dans $D$. En effet, nous pourrions sinon construire un ensemble bien ordonné ayant $\tilde{z}_{0}$ comme point d'accumulation. Ceci contredirait la proposition 2.3 puisque $\tilde{z}_{0}$ n'est pas de torsion nulle. Si lors de la modification de $\sigma$ et $\gamma, r$ a été choisi assez petit, $\widetilde{G}$ n'a donc pas de point fixe sur $\hat{\sigma} \backslash \sigma$.

Il est alors facile de voir que $\widetilde{G}$ n'a aucun fixe sur $\hat{\sigma}$ :

- $\hat{B}$ est disjoint de $D_{s}\left(\tilde{z}_{0}\right)$ et de $D_{i}\left(\tilde{z}_{0}^{\prime}\right)$. Il n'y a donc pas de point fixe sur les arcs contenus dans ces demi-droites.

- $\hat{D}$ est disjoint de $D_{i}\left(\tilde{z}_{0}\right)$ et de $D_{s}\left(\tilde{z}_{0}^{\prime}\right)$. Il n'y a donc pas de point fixe sur les arcs de $\sigma$ de la forme $\widetilde{G}^{-1}(I)$ où $I$ est un intervalle de $D_{i}\left(\tilde{z}_{0}\right)$ ou de $D_{s}\left(\tilde{z}_{0}\right)$.

d) On calcule maintenant l'indice Indice $(\widetilde{G}, \hat{\sigma})$ de $\widetilde{G}$ le long de $\hat{\sigma}$. On paramètre $\hat{\sigma}$ et $\hat{\gamma}$ par $\mathbb{T}^{1}$ de sorte que pour tout $t \in \mathbb{T}^{1}$,

$$
\widetilde{G}(\hat{\sigma}(t))=\hat{\gamma}(t)
$$

TOME $131-2003-\mathrm{N}^{\mathrm{O}} 1$ 


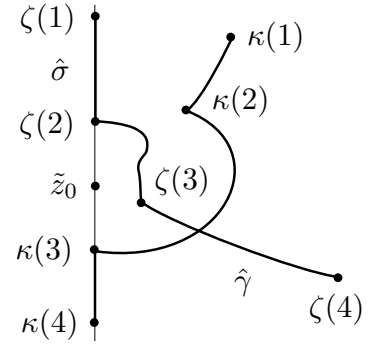

(a) $\hat{\sigma}$ et $\hat{\gamma}$ au voisinage de $\tilde{z}_{0}$

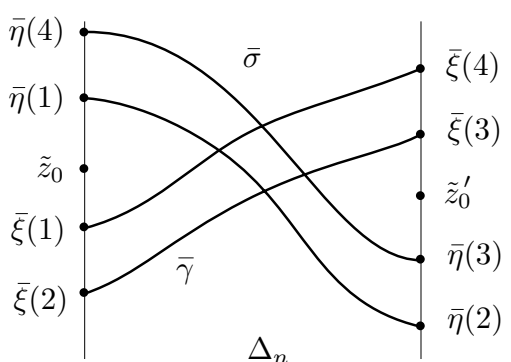

(b) Calcul de l'indice

FIGURE 7

Par définition, Indice $(\widetilde{G}, \hat{\sigma})$ est l'indice du champ de vecteurs $(\hat{\gamma}-\hat{\sigma})$ le long de $\hat{\sigma}$. Si l'on déforme continûment les courbes $\hat{\sigma}$ et $\hat{\gamma}$, sans annuler $\hat{\gamma}-\hat{\sigma}$, l'indice Indice $(\hat{\gamma}-\hat{\sigma}, \hat{\sigma})$ ne change pas. Les composantes de $\hat{\sigma} \cap \operatorname{Int}\left(\Delta_{0}\right)$ disjointes de $\sigma^{+}$et $\sigma^{-}$, de la forme $\widetilde{G}^{-1}(I)$ avec $I \subset D_{i}\left(\tilde{z}_{0}\right) \cup D_{s}\left(\tilde{z}_{0}\right)$ peuvent être rétractées à extrêmités fixées sur un arc de $D_{s}\left(\tilde{z}_{0}\right) \cup D_{i}\left(\tilde{z}_{0}^{\prime}\right)$ dans $\widetilde{\mathbb{A}} \backslash I$. On ne modifie pas l'arc $I$ correspondant de $\hat{\gamma}$. On procède ensuite de la même façon avec les composantes de $\hat{\gamma} \cap \operatorname{Int}\left(\Delta_{0}\right)$ disjointes de $\gamma^{+}$et $\gamma^{-}$.

Dans le cas où $D$ a été modifié près de $\tilde{z}_{0}, \hat{\sigma}$ est localement une réunion

$$
(\zeta(1), \zeta(2)) \cup[\zeta(2), \zeta(3)] \cup(\zeta(3), \zeta(4))
$$

où $(\zeta(1), \zeta(2))$, est un segment de $D_{s}\left(\tilde{z}_{0}\right)$. On note

$$
(\kappa(1), \kappa(2)) \cup[\kappa(2), \kappa(3)] \cup(\kappa(3), \kappa(4))
$$

la partition correspondante qui décrit $\hat{\gamma}$ au voisinage de $\tilde{z}_{0} \cdot(\kappa(3), \kappa(4))$ est un segment de $D_{i}\left(\tilde{z}_{0}\right)$ (voir figure 3.3 ). La dynamique locale de $\widetilde{G}$ en $\tilde{z}_{0}$ est approchée par la différentielle $\mathrm{D} \widetilde{G}\left(\tilde{z}_{0}\right)$. Par ce qui précède, $\tilde{z}_{0}$ a un nombre de torsion dans $\left[-\frac{1}{2}, 0[\right.$. On vérifie, que l'on peut modifier continûment les paramétrages de $\hat{\sigma}$ et $\hat{\gamma}$ sans annuler $\hat{\gamma}-\hat{\sigma}$ de sorte que les intervalles $(\zeta(1), \zeta(2))$ et $(\zeta(2), \zeta(4))$ correspondent aux intervalles $(\kappa(1), \kappa(3))$ et $(\kappa(3), \kappa(4))$. Il suffit de montrer que si $\left[\hat{\sigma}(t), \hat{\sigma}\left(t^{\prime}\right)\right]_{\hat{\sigma}}$ est un sous-arc de $[\zeta(2), \zeta(3)]_{\hat{\sigma}}$, alors les points $\hat{\sigma}(t)$ et $\hat{\gamma}\left(t^{\prime}\right)$ sont distincts. D'une part, d'après la forme de $\mathrm{D} \widetilde{G}\left(\tilde{z}_{0}\right)$, on peut relever $\operatorname{Angle}(\hat{\sigma}(t), \hat{\gamma}(t))$ dans $\left[-\frac{1}{2}, 0[\right.$; d'autre part, par définition de l'arc $[\kappa(2), \kappa(3)] \hat{\gamma}$, l'angle Angle $\left(\hat{\gamma}(t), \hat{\gamma}\left(t^{\prime}\right)\right)$ se relève également dans $\left[-\frac{1}{2}, 0[\right.$. Ainsi,

$$
-\frac{1}{2}<\operatorname{Angle}\left(\hat{\sigma}(t), \hat{\gamma}\left(t^{\prime}\right)\right)<0
$$

et donc $\hat{\sigma}(t) \neq \gamma\left(t^{\prime}\right)$. On procède de même en $\tilde{z}_{0}^{\prime}$.

BUlletin DE LA SOCiÉtÉ MATHÉmATiQUe DE FRANCE 
Les courbes que l'on obtient s'écrivent à présent (voir figure 3.3) :

$$
\begin{aligned}
& \bar{\sigma}=(\bar{\eta}(1), \bar{\eta}(2)) \cup[\bar{\eta}(2), \bar{\eta}(3)] \cup(\bar{\eta}(3), \bar{\eta}(4)) \cup[\bar{\eta}(4), \bar{\eta}(1)], \\
& \bar{\gamma}=[\bar{\xi}(1), \bar{\xi}(2)] \cup(\bar{\xi}(2), \bar{\xi}(3)) \cup[\bar{\xi}(3), \bar{\xi}(4)] \cup(\bar{\xi}(4), \bar{\xi}(1)) .
\end{aligned}
$$

$[\bar{\eta}(4), \bar{\eta}(1)],[\bar{\eta}(2), \bar{\eta}(3)],[\bar{\xi}(1), \bar{\xi}(2)]$ et $[\bar{\xi}(3), \bar{\xi}(4)]$ sont des intervalles de $D_{s}\left(\tilde{z}_{0}\right)$, $D_{i}\left(\tilde{z}_{0}^{\prime}\right), D_{i}\left(\tilde{z}_{0}\right)$ et $D_{s}\left(\tilde{z}_{0}^{\prime}\right)$. Les points $\bar{\eta}(i)$ et $\bar{\xi}(i), i=1, \ldots, 4$ correspondent aux mêmes paramètres de $\mathbb{T}^{1}$. Les arcs $(\bar{\eta}(1), \bar{\eta}(2)),(\bar{\eta}(3), \bar{\eta}(4)),(\bar{\xi}(2), \bar{\xi}(3))$ et $(\bar{\xi}(4), \bar{\xi}(1))$ sont inclus dans $\operatorname{Int}\left(\Delta_{0}\right)$. On calcule aisément l'indice

$$
\text { Indice }(\bar{\gamma}-\bar{\sigma}, \bar{\sigma})=-1 \text {. }
$$

Fin de la preuve du lemme 3.1. - L'indice de $\widetilde{G}$ le long de $\hat{\sigma}$ est -1 . L'application $\widetilde{G}$ possède donc un point fixe $\tilde{z}_{0}^{\prime \prime}$ dans $\operatorname{Int}(\hat{B})$. Puisque $\operatorname{Int}(\hat{B})$ ne contient pas $\tilde{z}_{0}$ ou $\tilde{z}_{0}^{\prime}$, l'orbite de $\tilde{z}_{0}^{\prime \prime}$ est distincte de celles de $\tilde{z}_{0}$ ou $\tilde{z}_{0}^{\prime}$. Par construction pour tout $0 \leq k \leq q$,

$$
\tilde{p}_{1}\left(\tilde{z}_{k}\right)<\tilde{p}_{1}\left(\widetilde{F}^{k}\left(\tilde{z}_{0}^{\prime \prime}\right)\right)<\tilde{p}_{1}\left(\tilde{z}_{k}^{\prime}\right) .
$$

L'ensemble $\widetilde{\Xi} \cup \widetilde{\mathcal{O}}\left(z_{0}^{\prime \prime}\right)$ est donc bien ordonné.

\section{BIBLIOGRAPHIE}

[1] Angenent (S.) - The periodic orbits of an area preserving twist map, Commun. Math. Phys., t. 115 (1988), pp. 353-374.

[2] Aubry (S.) \& Le Daeron (P.) - The discrete Frenkel-Kontorova model and its extension, Physica D, t. 8 (1983), pp. 381-422.

[3] Chenciner (A.) - La dynamique au voisinage d'un point fixe elliptique conservatif : de Poincaré et Birkhoff à Aubry et Mather, in Sém. Bourbaki, Astérisque, vol. 121-122, Soc. Math. France, 1982, exposé 622, pp. 147-170.

[4] Crovisier (S.) - Nombre de rotation et dynamique faiblement hyperbolique, Thèse, Université Paris-Sud, 2001, $3^{\mathrm{e}}$ partie.

[5] _ Langues d'Arnol'd généralisées des applications de l'anneau déviant la verticale, C. R. Acad. Sci. Paris, Sér. I, t. 334 (2002), pp. 47-52.

[6] Doundy (R.) - Application du théorème des tores invariants, Thèse de $3^{\mathrm{e}}$ cycle, Université Paris VII, 1982.

[7] Hall (G.) - A topological version of a theorem of Mather on twist maps, Ergod. Th. \& Dynam. Sys., t. 4 (1984), pp. 585-603.

[8] Herman (M.) - Sur les courbes invariantes par les difféomorphismes de l'anneau, vol. 1, Astérisque, vol. 103-104, Soc. Math. France, 1983.

[9] KatoK (A.) - Some remarks on Birkhoff and Mather twist map theorem, Ergod. Th. \& Dynam. Sys., t. 2 (1982), pp. 185-194.

[10] Le Calvez (P.) - Propriétés générales des applications déviant la verticale, Bull. Soc. Math. France, t. 117 (1989), pp. 69-102.

TOME $131-2003-\mathrm{N}^{\mathrm{O}} 1$ 
[11] _ Propriétés dynamiques des difféomorphismes de l'anneau et du tore, Astérisque, vol. 204, Soc. Math. France, 1991.

[12] Le Calvez (P.) \& Yoccoz (J.-C.) - Un théorème d'indice pour les homéomorphismes du plan au voisinage d'un point fixe, Ann. of Math., t. 146 (1997), pp. 241-293.

[13] Mather (J.) - Existence of quasi-periodic orbits for twist homeomorphisms of the annulus, Topology, t. 21 (1982), pp. 457-467.

[14] - Amount of rotation about a point and the Morse index, Commun. Math. Phys., t. 94 (1984), pp. 141-153. 